\title{
Ultrathin Polymer Membranes with Patterned, Micrometric Pores for Organs-on-chips
}

\author{
Virginia Pensabene ${ }^{\star}, 1,2,3$, Lino Costa ${ }^{4}$, Alexander Terekhov ${ }^{4}$, Juan S. Gnecco ${ }^{5}$, John \\ Wikswo $^{1,6,7,8}$, and William Hofmeister ${ }^{4,6}$ \\ 1 Department of Biomedical Engineering; Vanderbilt University, Nashville, TN 37235 USA \\ ${ }^{2}$ School of Electronic and Electrical Engineering, University of Leeds, Leeds, LS2 9JT, UK. \\ ${ }^{3}$ School of Medicine, Leeds Institute of Biomedical and Clinical Sciences, University of Leeds, \\ Leeds, LS9 7TF, UK. \\ ${ }^{4}$ Center for Laser Applications, University of Tennessee Space Institute, Tullahoma, TN 37388 \\ USA \\ ${ }^{5}$ Department of Cellular and Molecular Pathology, Vanderbilt University, Nashville, TN 37235 \\ USA \\ 6 Vanderbilt Institute for Integrative Biosystems Research and Education; Vanderbilt University, \\ Nashville, TN 37235 USA \\ 7 Department of Physics and Astronomy; Vanderbilt University, Nashville, TN 37235 USA \\ ${ }^{8}$ Department of Molecular Physiology and Biophysics; Vanderbilt University, Nashville, TN 37235 \\ USA
}

\section{Abstract}

The basal lamina or basement membrane (BM) is a key physiological system that participates in physicochemical signaling between tissue types. Its formation and function are essential in tissue maintenance, growth, angiogenesis, disease progression, and immunology. In vitro models of the BM, e.g., Boyden and transwell chambers, are common in cell biology and lab-on-a-chip devices where cells require apical and basolateral polarization. Extravasation, intravasation, membrane transport of chemokines, cytokines, chemotaxis of cells, and other key functions are routinely studied in these models. The goal of the present study was to integrate a semipermeable ultrathin polymer membrane with precisely positioned pores of $2 \mu \mathrm{m}$ diameter in a microfluidic device with apical and basolateral chambers. We selected poly (L-lactic acid) (PLLA), a transparent biocompatible polymer, to prepare the semipermeable ultrathin membranes. The pores were

\footnotetext{
*Corresponding Author Virginia Pensabene, virginia.pensabene@ vanderbilt.edu, Vanderbilt University C1136 Medical Center North, Nashville, TN 37235, (615)-343-8978. New address: V.Pensabene@leeds.ac.uk, School of Electronic and Electrical Engineering, University of Leeds, Leeds, LS2 9JT, UK, +44 (0) 7713371521.

Author Contributions

The manuscript was written through contributions of all authors. All authors have given approval to the final version of the manuscript. ASSOCIATED CONTENT

Supporting Information. This section provides additional images of PVA replicas coated with PLLA and closer view of the PVA needles after coating. Additionally, the results of the permeability measurements are summarized. This material is available free of charge via the Internet at http://pubs.acs.org.
} 
generated by pattern transfer using a three-step method coupling femtosecond laser machining, polymer replication, and spin coating. Each step of the fabrication process was characterized by scanning electron microscopy to investigate reliability of the process and fidelity of pattern transfer.

In order to evaluate the compatibility of the fabrication method with organs-on-a-chip technology, porous PLLA membranes were embedded in polydimethylsiloxane (PDMS) microfluidic devices and used to grow human umbilical vein endothelial cells (HUVECS) on top of the membrane with perfusion through the basolateral chamber. Viability of cells, optical transparency of membranes and strong adhesion of PLLA to PDMS were observed, thus confirming the suitability of the prepared membranes for use in organs-on-a-chip devices.

\section{Graphical Abstract}

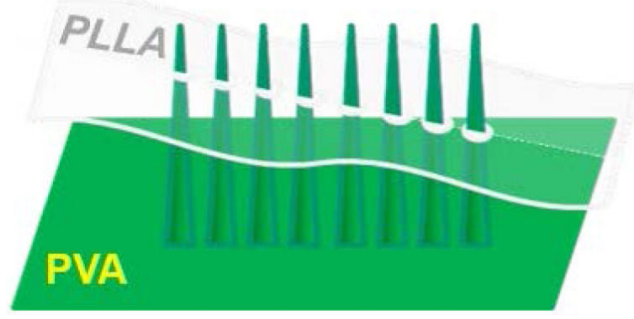

\section{Keywords}

Semipermeable ultrathin polymer membranes; microporous ultrathin polymer films; spin coating; microneedles; femtosecond laser machining; polymer replication

\section{INTRODUCTION}

In vitro models are an essential part of cell biology. Transwells have been widely adopted for polarized cell and migration assays since Boyden's initial chemotaxis experiment. ${ }^{1}$ The promise of organs-on-a-chip technology is essentially to create a more robust model of the complex electrophysicochemical systems that control cell function and fate. ${ }^{2,3}$ In static transwells as well as complex microfluidic organ models, a key element is the basil lamina or basement membrane (BM). ${ }^{4}$ This membrane supports diverse epithelial cell types and participates in signaling and transport for the system. ${ }^{5}$ In contrast to the static transwell and Boyden chamber devices, microfluidic barrier devices offer the possibility of using physiologically realistic flow of cell culture media so that the resulting shear stresses polarize the endothelial cells on the vascular side of the BM, as might be found in organ-onchip models of the blood-brain barrier. ${ }^{6,7}$ Critical properties of a semipermeable membrane in a microfluidic device include controlled porosity, high species flux, mechanical strength, surface biocompatibility, and optical transparency. Polycarbonate and polyester membranes are the main materials for transwell cell culture and diffusion experiments. These membranes are far thicker than the BM and the transparency is not optimal in bright field light, confounding observation by differential interference contrast. Ultrathin polymer films 
(nanofilms) are promising candidates for the membrane model system. They are closer in thickness to native BM, are optically transparent and compatible with cell culture.

Nanofilms with thicknesses ranging from tens to hundreds of nanometers belong to an interesting class of polymeric nanomaterials having lateral-dimension-to-thickness ratios that are greater than $10^{6}$, and unique thickness-dependent interfacial and mechanical properties. In particular, previous studies have revealed that such films can have noncovalent high adhesiveness to surfaces, tunable flexibility and molecular permeability, ${ }^{8}$ defined structural color, ${ }^{9}$ and mechanical strength, ${ }^{10}$ and possibly conductive ${ }^{11}$ and magnetic properties. ${ }^{12}$ These characteristics make such films a promising solution for multiple applications in the biomedical field. As an example, thanks to their quasi-twodimensional nature, such nanofilms resemble the lamina basalis of the extracellular matrix in human tissues, the ideal structure to direct the cellular organization and function thereby enabling organ regeneration and function.

Nanofilm fabrication techniques are diverse, and include layer-by-layer, ${ }^{13}$ LangmuirBlodgett methods ${ }^{14}$ and others. The simplest route to fabricate freestanding and easy-tohandle nanofilms is spin coating from a liquid polymer-solvent solution. ${ }^{15}$ In previous works, full characterization of plain nanofilm structures was performed. The adhesion and proliferation of different cell types on poly (lactic acid) (PLLA) nanofilms ${ }^{16}$ and polyelectrolytic films ${ }^{17}$ was confirmed. We now report on the fabrication and characterization of ultrathin semipermeable PLLA membranes for organs-on-a-chip applications. Herein "semipermeable membranes" refer to membranes with ordered pores of a specific size, distribution and shape that are precisely patterned to allow passage of chemical species and cells of a specific size range, while blocking migration of other cells. The semipermeable membranes function as support for growth of cell layers and as separation between distinct microfluidic chambers.

Existing examples of organs-on-a-chip have used perforated polydimethylsiloxane (PDMS) membranes, ${ }^{18,19}$ polycarbonate ${ }^{7,20}$ or photoresist membranes. ${ }^{21,22,23}$ While the PDMS membranes have the advantages of optical transparency, tunable elastic modulus and can be easily integrated in a monolithic PDMS device, it is still difficult to reduce the thickness of the membrane below $5 \mu \mathrm{m}$. Submicron membrane thickness is essential for physical contact and paracrine communication between cells growing on both sides.

The engineered semipermeable ultrathin polymer membranes are an advancement over current solutions. Numerous approaches have been used to fabricate,${ }^{24}$, organic semipermeable membranes, ${ }^{25}$ such as polymer synthesis ${ }^{26}$, ion track etching of polymers ${ }^{27}$ and polymer phase separation combined with selective solvent etching ${ }^{28}$. However, most of these methods have drawbacks, mainly related to random arrangement of pores with a wide size distribution or nanometric/sub-micrometric pore size. In the assembly of a microfluidic device in PDMS, the different microfabricated layers are aligned to form the final microfluidic circuitry. Bonding of these layers with the membrane, for example by plasma activation of the surfaces, is efficient only when the surfaces are flat and regular. The presence of pores in correspondence with edges of the microfluidic channels makes instead the bonding unattainable.

ACS Appl Mater Interfaces. Author manuscript; available in PMC 2017 August 31. 
Other methods, such as laser perforation ${ }^{\mathbf{2 9 - 3 1}}$, proton beam writing ${ }^{\mathbf{3 2}}$, and focused ion beam milling ${ }^{33-36}$, allow direct fabrication of micro- and nanopores on an otherwise unpatterned polymer sheet. This approach offers precise control over the design and arrangement of the pores, but at the cost of relatively long processing times required for serial, pore-by-pore, fabrication of each membrane. Issues with localized melting, chemical degradation, and wrinkling of the polymer membrane around the opened pores, as well as redeposition of debris on membrane surface and implantation of $\mathrm{Ga}$, have been observed and reported for some of these direct fabrication methods in the cited literature.

Alternatively, ultrathin polymer membranes containing precisely patterned micrometric pores can be formed by spin coating assisted deposition of a polymer-solvent solution atop a sacrificial array of spatially ordered polyvinyl-alcohol (PVA) nanoneedles, followed by removal of the sacrificial PVA nanoneedle array. The PVA nanoneedles are formed by PVA replication of an array of surface nanometric pores formed on a fused silica wafer using femtosecond laser machining. The details and suitability of this novel approach are outlined herein for PLLA membranes.

\section{MATERIALS AND METHODS}

\section{Fabrication of fused silica molds}

As a first step towards creating the PVA needle array, a $10 \mathrm{~mm} \times 10 \mathrm{~mm}$ regular array of $201 \times 201$ pores was patterned on the surface of a $500 \mu \mathrm{m}$ thick UV grade fused silica wafer (Mark Optics, Inc.) using the single-pulse femtosecond laser machining technique first described by White, et al. ${ }^{37}$ In their 2008 paper, White and co-workers demonstrated that high numerical aperture single-pulse femtosecond laser machining can create uniquelyshaped pores at the surface of fused silica, exhibiting high aspect ratios, with depths that exceed $10 \mu \mathrm{m}$ and diameters below $200 \mathrm{~nm}$. In the present work, femtosecond laser machining was carried out using the system described by Rajput. ${ }^{38}$ A dry microscope objective, namely a Nikon CF Plan Achromat 79173, was used to focus the femtosecond laser beam on the surface of the fused silica wafer. Energy per pulse of $3.2 \mu \mathrm{J}$ was used to open each pore. The laser-patterned wafer was soaked first in a $5 \mathrm{M} \mathrm{KOH}$ solution at $80^{\circ} \mathrm{C}$ for 2 hours, and then in de-ionized water also at $80^{\circ} \mathrm{C}$ for another 2 hours, in order to remove any machining debris. The washed wafer was dried under a stream of nitrogen, and used to prepare PLLA membranes for characterization purposes.

A second fused silica mold, designed to prepare PLLA membranes for use in organs-on-achip trials was femtosecond laser machined in a similar way. This mold was patterned with a 16 row $\times 100$ column rectangular array of surface pores. The distance between rows was 400 $\mu \mathrm{m}$, while the distance between columns was $100 \mu \mathrm{m}$, thereby creating pores whose arrangement matched channels in the microfluidic device.

\section{Preparation of PVA nanoneedle arrays}

Once the femtosecond laser patterned fused silica mold was fabricated, the freestanding polymer nanoneedle arrays were produced using the novel solution-casting mold-replication method recently reported by Rajput and co-workers. ${ }^{39,40}$ In the present work, arrays of 
freestanding polyvinyl alcohol (PVA) nanoneedles (or PVA replicas) were prepared using the water/alcohol-based PVA mold release agent Partall® Film \#10 (Rexco). In particular, each fused silica mold containing an array of surface pores was coated with a thin layer of Partall@ Film \#10 using a foam paintbrush. The layer was exposed to a flow of nitrogen and allowed to dry (Fig.1a). The resulting $\sim 25 \mu \mathrm{m}$ thick PVA film with nanoneedles was peeledoff the fused silica mold and mounted on a $22 \times 22 \mathrm{~mm}^{2}$ glass or polyvinyl chloride (PVC) coverslip using double-sided adhesive tape (Fig. 1b).

\section{Preparation of PLLA membranes}

Spin-coating-assisted deposition of PLLA on the PVA replicas was performed using the same processing parameter values used to prepare plain $\sim 100 \mathrm{~nm}$ thin PLLA films reported in previous work ${ }^{41}$. A $10 \mathrm{mg} / \mathrm{mL}$ solution of PLLA in dichloromethane (molecular weight $60 \mathrm{kDa}$; Polysciences, Inc.) was applied to the PVA nanoneedle array and spun at $3000 \mathrm{rpm}$ for $30 \mathrm{~s}$ inside a Class 100 clean room (Fig. 1c). The resulting PLLA films were allowed to dry for 1 minute at $80^{\circ} \mathrm{C}$ and stored in polystyrene Petri dishes.

\section{Assembly of microfluidic devices}

A simple two-compartment device was assembled to test the semipermeable PLLA membranes in an organs-on-a-chip assembly. The device consists of two microfabricated layers in polydimethylsiloxane (PDMS, Sylgard® 184 silicone elastomer kit from Dow Corning, MI, USA), produced by SU8 softlithography ${ }^{42}$. Briefly, the bottom layer consists of 16 parallel channels ( $200 \mu \mathrm{m}$ width, $100 \mathrm{~mm}$ height, $10 \mathrm{~mm}$ length, and separated by a $200 \mu \mathrm{m}$ space), obtained by casting and curing liquid PDMS (10:1) on SU8-2100 mold (from Microchem, MA, USA) fabricated in a Class 100 clean room. The upper chamber was prepared by punching a thick $3 \mathrm{~mm}$ layer of PDMS with a sterile disposable $6 \mathrm{~mm}$ diameter punch. Ports to access the microfluidic channels were opened by punching $1.59 \mathrm{~mm}$ (1/16 inch) diameter holes.

In order to bond a PLLA membrane between the two PDMS layers, the channeled PDMS layer was gently pressed against the PLLA layer formed atop a PVA replica (Fig. 1d). These two pieces were then immersed in de-ionized water for 6 to 12 hours to allow water to gradually dissolve and remove the sacrificial PVA template, leaving behind a semipermeable ultrathin PLLA membrane with precisely patterned micrometer scale pores (Fig. 1e). The resulting PLLA membrane remained adherent to the channeled PDMS layer. The upper PDMS layer chamber and the PLLA membrane were then oxygen-plasma treated (600 mTorr, $100 \mathrm{~W}$, for $45 \mathrm{sec}$ ) and finally bonded together (Fig. 1f). Oxygen-plasma treatment renders the exposed surfaces hydrophilic. Once bonded the devices were immediately filled with deionized sterile water and stored at $4^{\circ} \mathrm{C}$ until used.

\section{Cell culture, staining and assays}

Human umbilical vein endothelial cells (HUVECS) ${ }^{43}$ were isolated from umbilical cord obtained from a de-identified placenta collected from patients who underwent elective cesarean sections between 37 and 39 weeks of gestation. All procedures related to the consent and collection of tissues were approved by the Vanderbilt University Institutional Review Board. Cells were cultured in EBM-2 media supplemented with $10 \%$ of fetal bovine 
serum (Lonza, USA) and with 1\% antibiotics/antimycotics. Purity of the isolation was validated by morphological and immunofluorescent staining for CD31 (DAKO, USA) before loading in the devices. Cells were maintained at $37^{\circ} \mathrm{C}$ in a saturated humidity atmosphere containing $95 \%$ air $/ 5 \% \mathrm{CO}_{2}$, and they were sub-cultured before reaching $70 \%$ confluence (approximately every 2 days).

After tripsinization and centrifugation, cells were suspended in full medium $(2000$ cells $/ \mu \mathrm{L})$ and $50 \mu \mathrm{L}$ were injected in the device. Medium was refreshed in the device every 2 days. Cell growth on the PLLA nanofilm was monitored for 7 days as proof of principle.

Vitality was investigated after 7 days in culture by using NucBlue ${ }^{\circledR}$ Live ReadyProbes ${ }^{\circledR}$ Reagent and NucGreen $®$ Dead 488 ReadyProbes $®$ Reagent (Molecular Probes, R37605 and R37109). $20 \mu \mathrm{L}$ of staining solutions were added directly to cells in full media and incubated for 20 minutes. NucBlue ${ }^{\circledR}$ Live Cell Stain emits at $460 \mathrm{~nm}$ when bound to DNA while NucGreen® Dead 488 reagent is a membrane-impermeable stain DNA of dead cells (excitation/emission at 504/523 nm). Cellular cytoskeleton was visualized by F-actin staining using ActinGreen ${ }^{\mathrm{TM}} 488$ ReadyProbes ${ }^{\circledR}$ Reagent from Molecular Probes. Cells were prepared for staining by $4 \%$ paraformaldehyde fixation.

Cells were finally observed with an inverted fluorescent microscope (EVOS, FL Cell Imaging System) and with Image J software (Rasband, W.S., ImageJ, U. S. National Institutes of Health, Bethesda, Maryland, USA).

\section{Loading of microfluidic devices}

The devices were sterilized by UV irradiation for 30 minutes prior to loading with cell medium and cells. Mimicking the seeding protocol used for cell culture in traditional transwell inserts, the water was replaced with EBM-2 cell-culture media supplemented with $10 \%$ of fetal bovine serum (Lonza, USA), first loading the lower microfluidic channels and then the upper chamber. The nanofilm was not coated with any adhesive protein other than that in the media. The wet device was then enclosed in a Petri dish and equilibrated at $37^{\circ} \mathrm{C}$ for 12 hours inside the incubator.

\section{RESULTS}

\section{SEM characterization of fused silica molds and PVA replicas}

The surfaces of both the femtosecond laser patterned fused silica mold and the corresponding PVA replica were imaged using a JEOL 6320 field emission scanning electron microscope (SEM). Both samples were sputter-coated with a few nanometers of platinum for successful imaging. An image of a single-pulse femtosecond laser machined pore is shown in Figure 2a. Images of the PVA replica, at various magnifications, are shown in Figures 2b-d.

This laser processing technique was previously described and applied for the fabrication of arrays of micro- and nano-needles, as well as for milling nanoporous surfaces ${ }^{44-47}$. The laser machining time is short and the final pattern of holes is regular. The shape of the final holes in the PLLA film, as well as the section of the PVA needles is well defined, which enables to 
peel off and to detach the needles without deformations. Interestingly, few nanoneedles do not appear in the PVA replica, which can be explained as a synchronization issue in the femtosecond laser setup. When the laser miss-fires, the holes in silica will not formed, thus the PVA needle will not appear. An example of this missing needle is shown in Fig. 2.b.

\section{SEM characterization of PLLA membrane}

The effectiveness of the spin coating of PLLA on the PVA microneedle array is apparent in Fig. 3d: the PLLA solution spread and coated the full surface of the replica. Due to the high aspect ratio of the PVA needles and lack of wettability, the tips of the needles were not completely covered during the spin coating (Supporting information, Fig. S1). Based on SEM analysis of the PVA nanoneedles before and after PLLA spin coating deposition, the nanoneedles appear straight and not deformed by the deposition process or the centrifugal force. As shown in Fig. S1 of the Supporting Information, the PLLA covers the base of the needles and accumulates at the top of them, without damaging their structure.

In the fabrication of the PVA replica, we observed that few needles (less than 10\%) are not perfectly released during the demolding of the PVA from the silica mold: they thus look deformed, bent or are missing. These failures are due to the not homogeneous curing of the PVA inside the microfabricated silica and specifically inside the laser drilled holes.

An irregular PLLA coating of these imperfect nanoneedles thus caused failed nanopores in the PLLA.

The sacrificial layer PVA was dissolved by immersion in de-ionized water, and once removed, the PVA needle sites become open pores in the PLLA nanofilm membrane. While in this report, the PLLA film was transfer bonded to the PDMS, the hydrophobic PLLA film can be floated freestanding on the water surface for visualization (Fig. 3a). The patterned pores can be visualized in bright field once the film is collected and dried on a flat surface, such as a silicon wafer or a glass coverslip (Fig. 3b-c). The size of the holes was measured by analysis of SEM images of 5 PLLA nanofilms. All the images were obtained at 350x magnification, as in Fig. 4-c. The diameter of the holes is $1,858 \mu \mathrm{m} \pm 0.33$. The PLLA nanofilm replicates perfectly the surface morphology and any irregularity of the PVA replica, including the features imposed by the laser in the original silica mold (Fig. 3d).

Different views of a PLLA semipermeable membrane bonded to the channeled, bottom PDMS layer are shown in Fig. 4.

In this case, the PLLA membrane adheres and permanently bonds to the PDMS. In fact, the sub micrometric thickness and the high aspect ratio (surface/thickness) facilitate the contact between the nanofilm and a second surface (AFM thickness measurements reported in Supporting materials Fig. S3).

Even if surface effects influence the mechanism of adhesion and can drastically change the adhesive properties, the adhesion is influenced by the elasticity of the films and the nanometric thickness as described in $^{48}$. Without taking in consideration the specific materials, the adhesion is mainly due to hydrogen bonding and Van der Wall's forces. ${ }^{49}$ 
In this work, PLLA was selected because of its degradation properties. The hydrolytic degradation of poly lactide is well documented in literature ${ }^{50-52}$ and degradation time has been correlated with molecular weight and production methods. Specifically in this work a low molecular weight (80-100 kDa), L-lactic acid (2-hydroxy propionic acid), produced in small batches ${ }^{53}$ with limited D-content was preferred to control the degradation time.

Furthermore, PLLA has a slow degradation rate compared to other commonly used polymers, such as poly( $\epsilon$-caprolactone) or polyethylene glycol: degradation of PLLA membranes with micrometric thickness in distilled water, Ringer's solution and pancreatin solution have been measured and reported in details ${ }^{54}$.

\section{Device Assembly}

The PLLA membrane bonded wrinkle-free to the channeled PDMS layer (Fig. 4a). The sixteen rows of micrometric pores of the PLLA membrane aligned precisely above the sixteen-microfluidic channels. The sacrificial PVA layer provides a means of handling the nanofilm and mounting the film on the channeled PDMS layer preventing damage to the film; a key concern in manipulation of the ultrathin membrane.

Since both PDMS and PLLA are optically transparent, alignment of various components was done under a stereomicroscope. Together with the pattern of surface pores, the original silica mold has femtosecond laser machined surface alignment marks and a lateral identification label (Fig. 4.b-d); these microscopic features transfer to the PVA and PLLA films and serve as alignment guides during device assembly.

We observed that the PLLA membrane adhered tightly to the channeled PDMS layer and did not collapse inside the channels (Fig. 5a): while loading with two different food color solutions the upper (blue) and lower (red) chambers, the liquids filled up the empty channels and leakage was not observed between the film and the PDMS layers. The two solutions appeared confined in the respective microfluidic compartments, and started to mix only one hour later. Cells were then seeded directly on the PLLA membrane in the upper chamber without prior coating of the surface. Approximately 100,000 cells were seeded at Day 0 in the device, filling the upper chamber without leakage between the layers. It was possible to observe the cell behavior and monitor growth by optical microscopy due to the transparency of the PLLA membrane in bright field.

Oxygen plasma treatment did not adversely affect the biological properties of the PLLA film surfaces; the grow rate and cell morphology of the cells on PLLA inside the device were comparable to the control flask (see Fig. S5 in Supporting Material). As shown in Fig. 5c-d, cells proliferate rapidly in the device and with very low mortality (below 1\%). The media of the two chambers was replaced every 48 hours and after 7 days the adhesion of the cells on the semipermeable PLLA membrane was confirmed by F-actin staining (Fig. 5.d). Cells were trypsinazed and the suspensions from the two chambers were collected to measure the amount of cells by using a cell counter. Cells were found only in medium from the upper chamber, thus proving that the size of the pores did not exceed the nominal value of $2 \mu \mathrm{m}$ in diameter (data not shown). At the same time, the permeability of the membrane was 
confirmed by $150 \mathrm{kDa}$ FITC-dextran diffusion measurements (as reported in Supporting information, Figure S2).

PLLA is approved by Food and Drug Administration as biocompatible material for in vitro and in vivo use, and it has been reported in several publications as material for tissue engineering and scaffold. ${ }^{55-58}$ The chemical biocompatibility of PLLA nanofilms has been widely characterized in vitro with different cell types ${ }^{12,16}$, and assessed with in vivo animal model $^{8,41,55}$.

The small volume of the nanofilm $\left(\sim 10^{-4} \mu \mathrm{L}\right)$ limits the choice of possible techniques to evaluate the chemical biocompatibility with the cell culture media and to quantify the absorption of molecules from the media into the PLLA film. Future work will be dedicated to the analysis of the composition of cell culture media after long term contact with the PLLA.

\section{DISCUSSION AND CONCLUSIONS}

Fabrication of biocompatible, transparent, semipermeable ultrathin polymer membranes with precisely patterned micropores is difficult by perforation using e-beam lithography, focused ion beam milling, or laser micromachining. Additionally, such processes lack production scalability. Forming membranes using a molding approach is also difficult to implement. First of all, the polymer melt, polymer precursor, or the polymer-solvent solution being used must have sufficiently low viscosity to spread and fill the surface of the mold completely and homogeneously, without leaving a residual layer on top of the mold micropillars. Mechanical peeling of the membrane is problematic due to the thickness of the membrane. Once released from the mold, the membrane must be transferred and precisely attached to the microfluidic device and without any wrinkles and folds.

The method reported in this paper provides a quick, repeatable and convenient route to prepare multiple copies of the desired membranes. First, the direct one-step femtosecond laser machining of the fused silica molds can be carried out at kilohertz rates. With the current system, this translates to patterning a regular array of four million surface pores inside a one square centimeter area in less than one hour. Molds can be prepared with a distance between pores as small as $2 \mu \mathrm{m}$. The construction of the silica mold with alignment marks allows the user to control the size and location of each pore, and more importantly, the density of pores. While in this demonstration, each microfluidic channel had a single line of 100 pores each separated by $100 \mu \mathrm{m}$, the ability to separate pores by as little as $2 \mu \mathrm{m}$ would allow us to have tens of thousands of pores in each channel. The ability to control the location of pores means that the femtosecond laser drilling need not be wasted on regions of membrane sandwiched between solid PDMS, and the presence of pores and any associated surface irregularities is avoided in such regions where PDMS bonding is critical to avoids leaks and channel cross-talk.

Chemically inert and mechanically hard, the fused silica mold can be used to prepare thousands of PVA replicas. The PVA formulation used does not stick to fused silica. Each PVA replica precisely and repeatedly duplicates all the features of the mold, yielding 
straight, standing, and aligned high aspect-ratio nanoneedles. PVA replicas can be produced relatively quickly (15-30 minutes) and the mold can be easily cleaned from residual traces of PVA by hot water. The water-solubility and organic solvent-resistance of PVA makes it a suitable sacrificial material to use as template in preparing semipermeable membranes for many organic solvent-soluble polymers of interest. Finally, the PVA template provides a convenient vehicle to transfer, align and attach the ultrathin membrane to the microfluidic device without introducing undesirable wrinkles or folds in the final assembly.

While the selection of biocompatible polymers is wide and the fabrication of films with micron thicknesses can be achieved with several methods, handling of a perforated thin membrane represents a technological challenge. In this work we selected PLLA since its biocompatibility is well demonstrated. Furthermore, the PVA sacrificial layer is fundamental for handling and positioning of the ultrathin membrane PLLA: while an unpatterned sacrificial layer is traditionally used to release positioning polymeric films from their support, for example in stretchable and epidermal electronics ${ }^{59,60}$, in this case the dissolvable PVA includes not only the needles but also temporary labels and frames to align the pattern of needles with the microfabricated channels in the PDMS.

The semipermeable property of these PLLA membrane guarantees the possibility to separate two fluidic compartments and to select the size of particulates (i.e., cells versus macromolecules) able to pass through the pores. This is a key characteristic in lab-on-a-chip technology, where cellular extravasation and passage of chemical species between communicating compartments need to be controlled. Organs-on-a-chip seeks to understand complex processes; e.g., organ development, embryogenesis, tumor metastasis, and leukocyte infiltration that are regulated by cellular responses to multiple (competing) chemokines as well as autocrine feedback loops, cell-cell interactions, and mechanical stress. The result outlined herein of a scalable, tunable semipermeable nanofilm membrane will contribute to the success of organs-on-a-chip models and enable more faithful recapitulation of in vivo conditions.

\title{
Supplementary Material
}

Refer to Web version on PubMed Central for supplementary material.

\section{ACKNOWLEDGMENT}

\author{
The authors thank Dr. Brian Canfield for help in developing the LabView code used to operate the femtosecond \\ laser micromachining facility, and Miss Michela Comune for her help with the characterization of the nanofilm. \\ Additionally, the authors acknowledge Prof. Anthony Hmelo for the use of the SEM microscope at the Vanderbilt \\ Institute of Nanoscale Science and Engineering. \\ Funding Sources \\ This work was supported by the Tennessee Higher Education Commission, through a grant to the Center for Laser \\ Applications, University of Tennessee Space Institute. \\ Research reported in this publication was supported in part by the National Center for Advancing Translational \\ Sciences of the NIH under Award Nos.UH2TR000491 and UH3TR000491 and the Vanderbilt Institute for \\ Integrative Biosystems Research and Education. The content is solely the responsibility of the authors and does not \\ necessarily represent the official views of the funding agencies and organizations.
}


ABBREVIATIONS

$\begin{array}{ll}\text { PLLA } & \text { poly (L-lactic acid) } \\ \text { PVA } & \text { polyvinyl-alcohol } \\ \text { BM } & \text { basement membrane } \\ \text { PDMS } & \text { polydimethylsiloxane } \\ \text { HUVECS } & \text { human umbilical vein endothelial cells } \\ \text { PVC } & \text { polyvinyl chloride }\end{array}$

\section{REFERENCES}

1. Boyden S. The Chemotactic Effect of Mixtures of Antibody and Antigen on Polymorphonuclear Leucocytes. J. Exp. Med. 1962; 115(3):453-466. [PubMed: 13872176]

2. Ingber DE. Reverse Engineering Human Pathophysiology with Organs-on-Chips. Cell. 2016; 164(6):1105-1109. [PubMed: 26967278]

3. Wikswo JP. The Relevance and Potential Roles of Microphysiological Systems in Biology and Medicine. Exp.Biol.Med. 2014; 2014; 239:1061-1072.

4. Huh D, Hamilton GA, Ingber DE. From 3D Cell Culture to Organs-on-chips Trends. Cell Biol. 2011; 21(12):745-754.

5. McCaffrey L, M. Macara IG. Epithelial Organization, Cell Polarity and Tumorigenesis Trends. Cell Biol. 2011; 21(12):727-735.

6. Griep LM, Wolbers F, de Wagenaar B, ter Braak PM, Weksler BB, Romero IA, Couraud PO, Vermes I, van der Meer AD, van den Berg A. BBB ON CHIP: Microfluidic Platform to Mechanically and Biochemically Modulate Blood-Brain Barrier Function. Biomed.Microdevices. 2013; 15(1):145-150. [PubMed: 22955726]

7. Brown JA, Pensabene V, Markov DA, Allwardt V, Neely MD, Shi M, Britt CM, Hoilett OS, Yang Q, Brewer BM, Samson PC, McCawley LJ, Webb DJ, Li D, Bowman AB, Reiserer RS, Wikswo JP. Recreating Blood-Brain barrier Physiology and Structure on Chip: A Novel Neurovascular Microfluidic Bioreactor. Biomicrofluidics. 2015; 9:0541241-05412415.

8. Okamura Y, Kabata K, Kinoshita M, Saitoh D, Takeoka S. Free-Standing Biodegradable Poly(lactic acid) Nanosheet for Sealing Operations in Surgery. Adv. Mater. 2009; 21(43):4388-4392. [PubMed: 26042950]

9. Fujie T, Okamura Y, Takeoka S. Selective Surface Modification of Free-Standing Polysaccharide Nanosheet with Micro/nano-particles Identified by Structural Color Changes. Colloids Surf., A. 2009; 334(1-3):28-33.

10. Markutsya S, Jiang CY, Pikus Y, Tsukruk VV. Freely Suspended Layer-by-layer Nanomembranes: Testing Micromechanical Properties. Adv. Funct. Mater. 2005; 15(5):771-780.

11. Zucca A, Yamagishi K, Fujie T, Takeoka S, Mattoli V, Greco F. Roll to Roll Processing of Ultraconformable Conducting Polymer Nanosheets. J. Mater. Chem. C. 2015; 3(25):6539-6548.

12. Taccola S, Desii A, Pensabene V, Fujie T, Saito A, Takeoka S, Dario P, Menciassi A, Mattoli V. Free-Standing Poly(L-lactic acid) Nanofilms Loaded with Superparamagnetic. Nanoparticles Langmuir. 2011; 27(9):5589-5595. [PubMed: 21456538]

13. Jiang CY, Tsukruk VV. Freestanding Nanostructures via Layer-by-layer Assembly. Adv. Mater. 2006; 18(7):829-840.

14. Endo H, Kado Y, Mitsuishi M, Miyashita T. Fabrication of Free-standing Hybrid Nanosheets Organized with Polymer Langmuir-Blodgett Films and Gold Nanoparticles. Macromolecules. 2006; 39(16):5559-5563.

15. Cho J, Char K, Hong JD, Lee KB. Fabrication of Highly Ordered Multilayer Films Using a Spin Self-assembly Method. Adv. Mater. 2001; 13(14):1076-1078. 
16. Ricotti L, Taccola S, Pensabene V, Mattoli V, Fujie T, Takeoka S, Menciassi A, Dario P. Adhesion and Proliferation of Skeletal Muscle Cells on Single Layer Poly(lactic Acid) Ultra-thin Films. Biomed. Microdevices. 2010; 12(5):809-819. [PubMed: 20552402]

17. Ricotti L, Taccola S, Bernardeschi I, Pensabene V, Dario P, Menciassi A. Quantification of Growth and Differentiation of C2C12 Skeletal Muscle Cells on PSSPAH-based Polyelectrolyte Layer-bylayer Nanofilms. Biomed. Mater. 2011; 6(3):031001. [PubMed: 21566276]

18. Kim HJ, Ingber DE. Gut-on-a-Chip Microenvironment Induces Human Intestinal Cells to Undergo Villus Differentiation. Integr. Biol. 2013; 5(9):1130-1140.

19. Kim HJ, Huh D, Hamilton G, Ingber DE. Human gut-on-a-chip Inhabited by Microbial Flora that Experiences Intestinal Peristalsis-like Motions and Flow. Lab on a Chip. 2012; 12(12):2165-2174. [PubMed: 22434367]

20. Booth R, Kim H. Characterization of a Microfluidic in vitro Model of the Blood-Brain Barrier (mu BBB). Lab on a Chip. 2012; 12(10):1784-1792. [PubMed: 22422217]

21. Esch MB, Sung JH, Yang J, Yu CH, Yu JJ, March JC, Shuler ML. On chip Porous Polymer Membranes for Integration of Gastrointestinal Tract Epithelium with Microfluidic 'Body-on-achip’ Devices. Biomed. Microdevices. 2012; 14(5):895-906. [PubMed: 22847474]

22. Ornoff DM, Wang Y, Allbritton NL. Characterization of Freestanding Photoresist Films for Biological and MEMS Applications. J.Micromech.Microeng. 2013; 23(2) Article 025009.

23. Kim MY, Li DJ, Pham LK, Wong BG, Hui EE. Microfabrication of High-Resolution Porous Membranes for Cell Culture. J.Membr.Sci. 2014; 452:460-469.

24. Agrawal AA, Nehilla BJ, Reisig KV, Gaborski TR, Fang DZ, Striemer CC, Fauchet PM, McGrath JL. Porous Nanocrystalline Silicon Membranes as Highly Permeable and Molecularly Thin Substrates for Cell Culture. Biomaterials. 2010; 31(20):5408-5417. [PubMed: 20398927]

25. Ulbricht M. Advanced Functional Polymer Membranes. Polymer. 2006; 47(7):2217-2262.

26. Uragami T, Naito Y, Sugihara M. Studies on Synthesis and Permeability of Special Polymer Membranes Permeation Characteristics and Structure of Polymer Blend Membranes from Poly(vinylidene Fluoride) and Poly(ethylene Glycol). Polym. Bull. 1981; 4(10):617-622.

27. Martin CR, Nishizawa M, Jirage K, Kang M. Investigations of the Transport Properties of Gold Nanotubule Membranes. J. Phys. Chem. B. 2001; 105(10):1925-1934.

28. Zhang H, Takeoka S. Morphological Evolution within Spin-cast Ultrathin Polymer Blend Films Clarified by a Freestanding Method. Macromolecules. 2012; 45(10):4315-4321.

29. Wang Z, Du Z, Chan JKY, Teoh SH, Thian ES, Hong M. Direct Laser Microperforation of Bioresponsive Surface-Patterned Films with Through-Hole Arrays for Vascular TissueEngineering Application ACS. Biomater. Sci. Eng. 2015; 1:1239-1249.

30. Kim TN, Campbell K, Groisman A, Kleinfeld D, Schaffer CB. Femtosecond Laser-Drilled Capillary Integrated into a Microfluidic Device. Appl. Phys. Lett. 2005; 86:201106.

31. Xia B, Jiang L, Li X, Yan X, Zhao W, Lu Y. High Aspect Ratio, High-Quality Microholes in PMMA: a Comparison Between Femtosecond Laser Drilling in Air and in Vacuum. Appl. Phys. A. 2015; 119:61-68.

32. Uchiya N, Uchiya N, Harada T, Murai M, Nishikawa H, Haga J, Sato T, Ishii Y, Kamiya T. Micromachining of Resists on Silicon by Proton Beam Writing. Nucl. Instrum. Methods Phys. Res., Sect. B. 2007; 260:405-408.

33. Kim S, Park MJ, Balsara NP, Liu G, Minor AM. Minimization of Focused Ion Beam Damage in Nanostructured Polymer Thin Films. Ultramicroscopy. 2011; 111:191-199. [PubMed: 21333856]

34. Orthacker A, Schmied R, Chernev B, Froch JE, Winkler R, Hobisch J, Trimmel G, Plank H. Chemical Degradation and Morphological Instabilities During Focused Ion Beam Prototyping of Polymers. Phys.Chem.Chem.Phys. 2014; 16:1658-1666. [PubMed: 24322544]

35. Schmied R, Froch JE, Orthacker A, Hobisch J, Trimmel G, Plank H. A Combined Approach to Predict Spatial Temperature Evolution and its Consequences during FIB Processing of Soft Matter. Phys.Chem.Chem.Phys. 2014; 16:6153-6158. [PubMed: 24562543]

36. Lee CC, Proust G, Alici G, Spinks GM, Cairney JM. Three-dimensional Nanofabrication of Polystyrene by Focused Ion Beam. J. Microsc. 2012; 248:2, 129-139. 
37. White YV, Li XX, Sikorski Z, Davis LM, Hofmeister W. Single-pulse Ultrafast-laser Machining of High Aspect Nano-holes at the Surface of $\mathrm{SiO}_{2}$. Optics Express. 2008; 16(19):14411-14420. [PubMed: 18794977]

38. Rajput D, Costa L, Lansford K, Terekhov A, Hofmeister W. Solution-Cast High-Aspect-Ratio Polymer Structures from Direct-Write Templates ACS. Appl. Mater. Interfaces. 2013; 5(1):1-5.

39. Optofluidics: Fundamentals, Devices, and Applications. McGraw-Hill; New York: New York: 2010.

40. Pensabene V, Patel PP, Williams P, Cooper TL, Kirkbride KC, Giorgio TD, Tulipan NB. Repairing Fetal Membranes with a Self-adhesive Ultrathin Polymeric Film: Evaluation in Mid-gestational Rabbit Model. Ann. Biomed. Eng. 2015; 43(8):1978-1988. [PubMed: 25549772]

41. Tang, SKY.; Whitesides, GM. Basic Microfluidic and Soft Lithographic Techniques, in Optofluidics: Fundamentals, Devices, and Applications. Fainman, Y.; Psaltis, D.; Yang, C., editors. 2010 The McGraw-Hill Companies, Inc.; p. 15Chapter 2 ISBN: 9780071601566

42. Baudin B, Bruneel A, Bosselut N, Vaubourdolle M. A Protocol for Isolation and Culture of Human Umbilical Vein Endothelial Cells. Nat. Protoc. 2007; 2(3):481-485. [PubMed: 17406610]

43. Hofmeister LH, Costa L, Balikov DA, Crowder SW, Terekhov A, Sung H-J, Hofmeister WH. Patterned Polymer Matrix Promotes Stemness and Cell-cell Interaction of Adult Stem Cells. J. Biol. Eng. 2015; 9:18. [PubMed: 26464581]

44. Zachman A, Hofmeister L, Costa L, Boire T, Hwang Y-S, Hofmeister W, Sung H-J. Femtosecond Laser-patterned Nanopore Arrays for Surface-mediated Peptide Treatment Nanomedicine. Nanotechnology, Biology and Medicine. 2014; 10:1, 11-14.

45. Rajput D, Crowder SW, Hofmeister L, Costa L, Sung H-J, Hofmeister W. Cell Interaction Study Method using Novel 3D Silica Nanoneedle Gradient. Arrays Colloids Surf., B. 2013; 102:111116.

46. Rajput D, Costa L, Terekhov A, Lansford K, Hofmeister W. Silica Coating of Polymer Nanowires produced via Nanoimprint Lithography from Femtosecond Laser Machined Templates. Nanotechnology. 2012; 23:10.

47. Fujie T, Matsutani N, Kinoshita M, Okamura Y, Saito A, Takeoka S. Adhesive, Flexible, and Robust Polysaccharide Nanosheets Integrated for Tissue-Defect Repair. Adv. Funct. Mater. 2009; 19:2560-2568.

48. Andrew Phoenix, David; Ahmed, Waqar; Fujie, T.; Takeoka, S., editors. Advances in Nanosheet Technology Towards Nanomedical Engineering. Vol. 73. One Central Press (OCP); Nanobiotechnology. ISBN (eBook): 978-1-910086-03-2 Chapter 3

49. Jiménez A, Peltzer M, Ruseckaite R. Poly(lactic acid) Science and Technology: Processing, Properties, Additives and Applications. Royal Society of Chemistry. 2014:290-308.

50. Auras, RA.; Lim, L-T.; Selke, SEM.; Tsuji, H. Poly(lactic acid): Synthesis, Structures, Properties, Processing, and Applications. John Wiley \& Sons; 2011.

51. Shasteen C, Bin Cho Y. Controlling Degradation Rate of Poly(lactic Acid) for its Biomedical Applications. Biomedical Engineering Letters. 2011; 1:163.

52. Höglund A, Odelius K, Albertsson A-C. Crucial Differences in the Hydrolytic Degradation between Industrial Polylactide and Laboratory-Scale Poly(l-lactide). ACS Appl Mater Interfaces. 2012; 4(5):2788-2793. 23. [PubMed: 22563747]

53. Wang Z, Wang S, Guidoin R, Marois Y, Zhang Z. In vitro Homogeneous and Heterogeneous Degradation of Poly( $\epsilon$-Caprolactone/ Polyethylene Glycol/L-lactide): The Absence of Autocatalysis and the Role of Enzymes. J. Biomed. Mater. Res. A. 2006; 79:6. [PubMed: 16741981]

54. Pensabene V, Taccola S, Ricotti L, Ciofani G, Menciassi A, Perut F, Salerno M, Dario P, Baldini N. Flexible Polymeric Ultrathin Film for Mesenchymal Stem Cells Differentiation. Acta Biomater. 2011; 7:2883-2891. [PubMed: 21421086]

55. Gunatillake PA, Adhikari R. Biodegradable Synthetic Polymers for Tissue Engineering. Eur. Cells Mater. 2003; 5:1-16.

56. Tian H,Z, Tang X, Zhuang X, Chen, Jing X. Biodegradable Synthetic Polymers: Preparation, Functionalization and Biomedical Application. Prog. Polym. Sci. 2012; 37:237-280. 
57. Rezwan K, Chen Q, Blaker J, Boccaccini AR. Biodegradable and Bioactive Porous PolymerInorganic Composite Scaffolds for Bone Tissue Engineering. Biomaterials. 2006; 27:3413-3431. [PubMed: 16504284]

58. Jeong JW, Yeo WH, Akhtar A, Norton JJS, Kwack YJ, Li S, Jung SY, Su YW, Lee W, Xia J, Cheng HY, Huang YG, Choi WS, Bretl T, Rogers JA. Materials and Optimized Designs for HumanMachine Interfaces Via Epidermal Electronics. Adv. Mater. 2013; 25(47):6839-6846. [PubMed: 24327417]

59. Zucca A, Cipriani C, Sudha, Tarantino S, Ricci D, Mattoli V, Greco F. Tattoo Conductive Polymer Nanosheets for Skin-Contact Applications. Adv. Healthcare Mater. 2015; 4(7):983-990. 
A

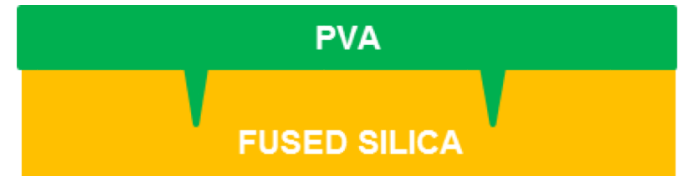

B

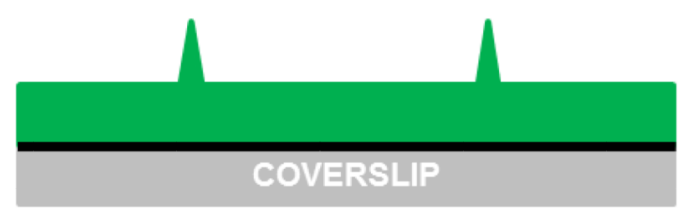

C

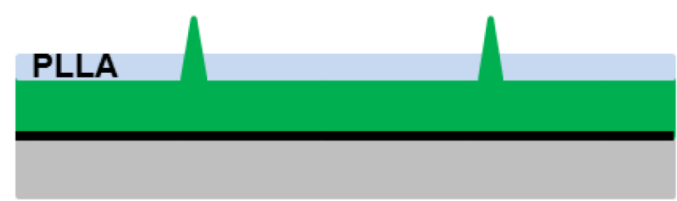

D

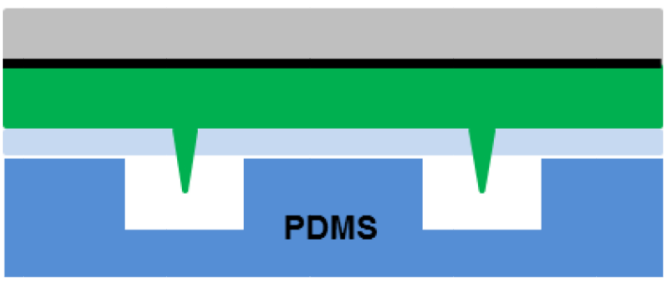

PLLA

$\mathbf{E}$

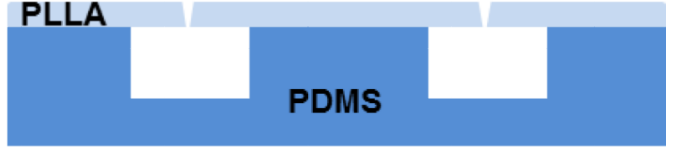

$\mathbf{F}$

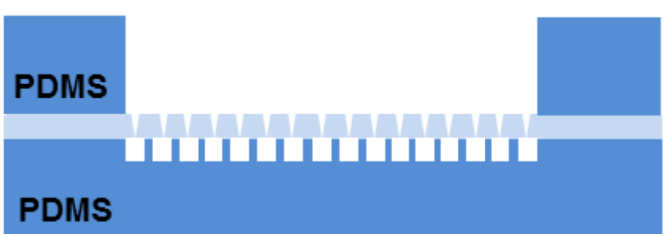

Fig. 1.

Process for fabrication of a semipermeable ultrathin PLLA membrane. a) PVA microneedle array is created by casting the PVA solution on a fused-silica mold and by allowing the PVA to dry. b) PVA replica is lift-off bonded to coverslip using adhesive tape (black solid line). c) PVA replica is spin coated with PLLA. d) PLLA film is placed in contact and aligned to the PDMS microfluidic layer. e) PLLA film is adherent on the PDMS layer. f) The final assembled device presents the PLLA barrier between two microfluidic chambers. 

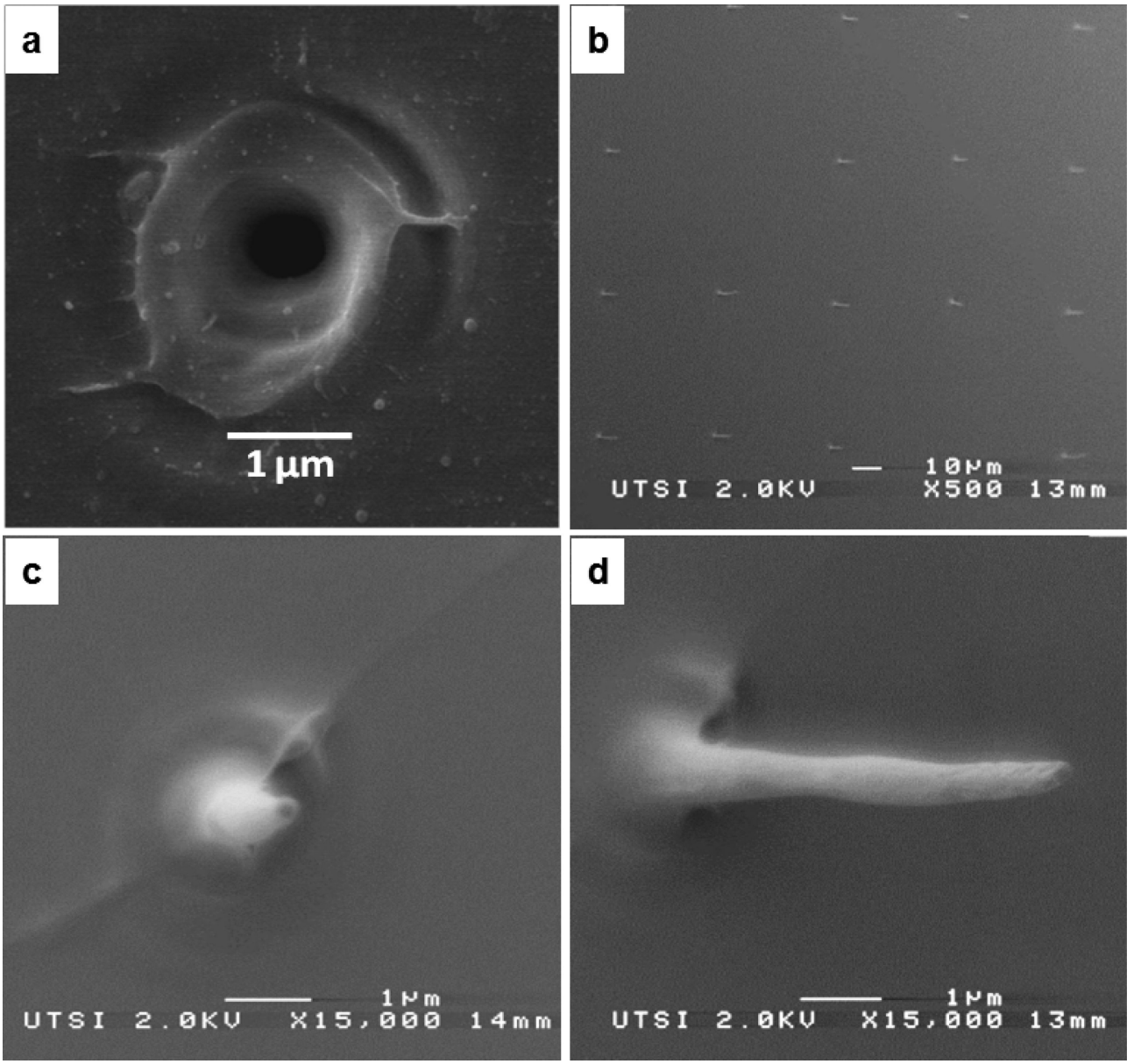

Figure 2. Process characterization

a) SEM image of a femtosecond laser machined pore on the surface of a fused silica wafer. The pore was opened using a single $3.2 \mu \mathrm{J}, 790 \eta \mathrm{m}, 160$ femtosecond laser pulse; b) SEM image of a PVA replica (Pt coated, $45^{\circ}$ stage tilt). c) A single PVA nanoneedle with a final length of $10 \mu \mathrm{m} . \mathrm{d})$ same PVA nanoneedle at $30^{\circ}$ stage tilt. 

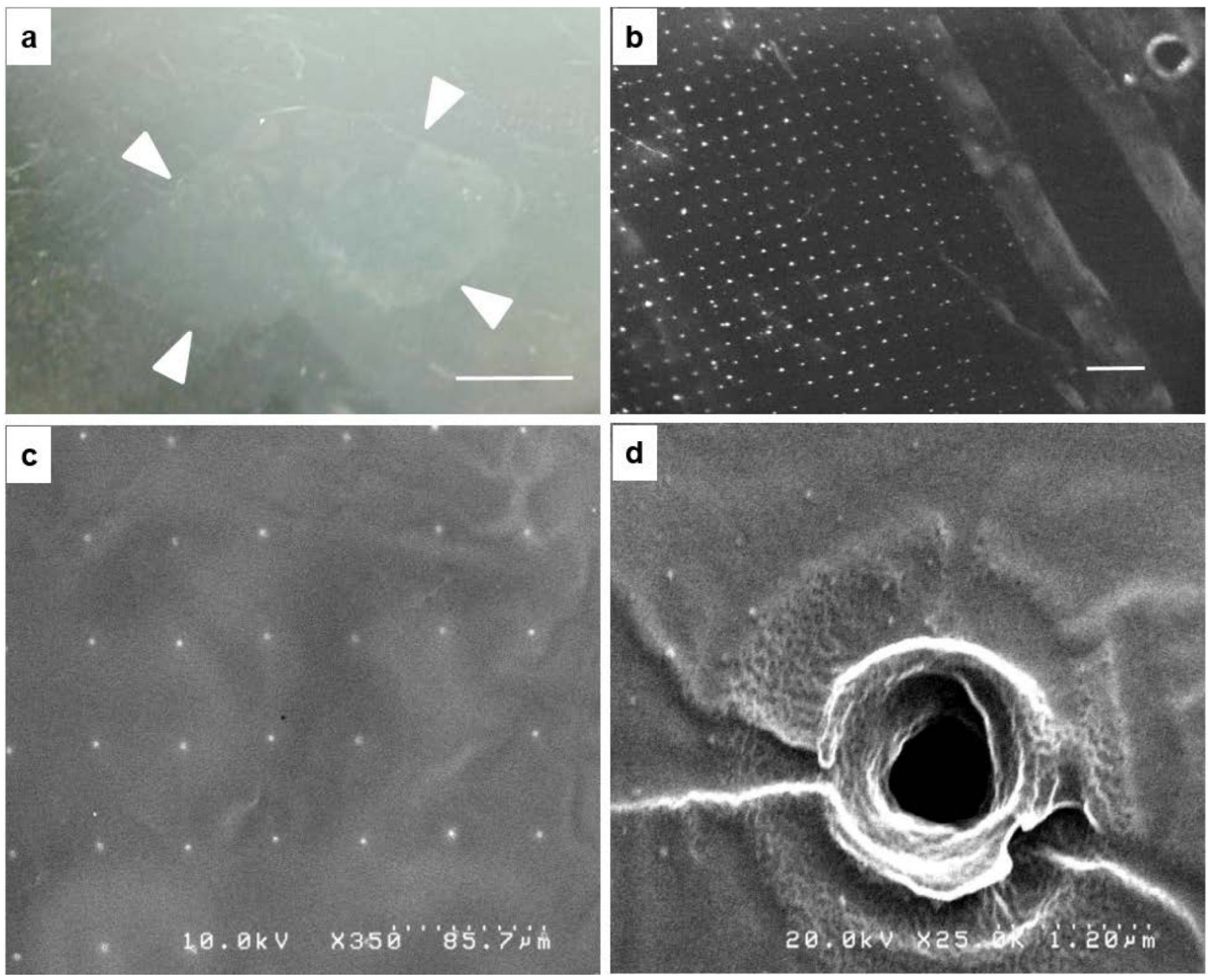

Fig. 3. Characterization of PLLA film

a) Film floating on water surface after the PVA template dissolved (film edges highlighted with white arrows, scale bar $1 \mathrm{~cm}$ ); b) optical microscope image of the film (holes appear as white dots, scale bar $500 \mu \mathrm{m}$ ); c) the array of holes in the film are visualized with the SEM ( $2 \mathrm{~nm}$ gold coating); d) A detail of a single hole in the PLLA film. 

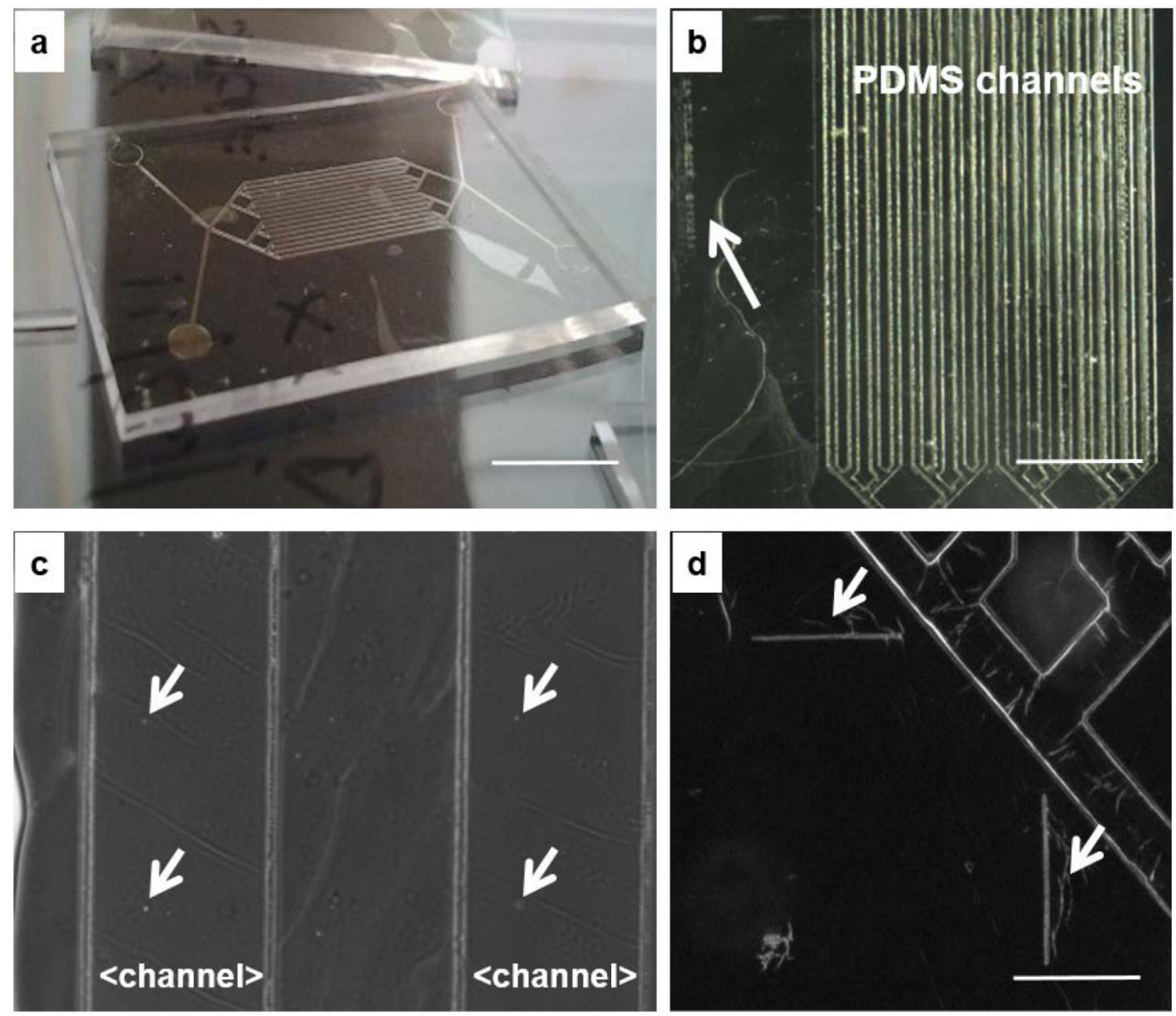

Fig. 4. Device assembly

a) PLLA film adherent to channeled, bottom PDMS layer (scale bar $1 \mathrm{~cm}$ ); b) PLLA film on a channeled PDMS bottom layer with arrow pointing to the lateral identification label (scale bar $4 \mathrm{~mm}$ ); c) magnified view of Figure $4 \mathrm{~b}$ showing a group of four micrometric pores in the PLLA membrane laying above the $200 \mu \mathrm{m}$-wide microfluidic channels in the PDMS layer; d) alignment features (scale bar $500 \mu \mathrm{m}$ ). 

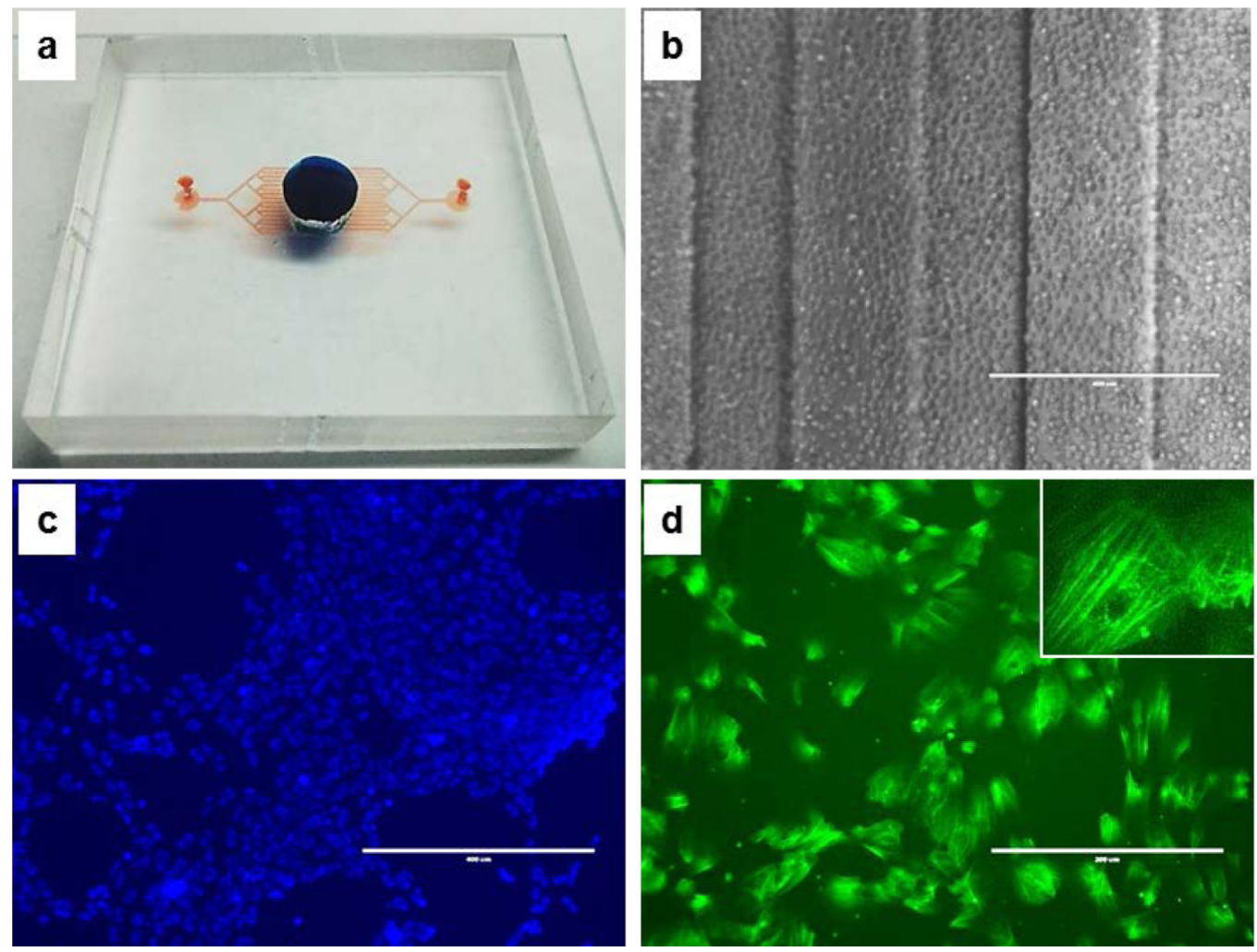

Fig. 5. Cells adhesion in the device

a) Full device (blue dye for upper chamber volume, red dye for the lower, channeled chamber volume); b) HUVECs at the time of loading (D0, 4x, ph, scale bar $400 \mu \mathrm{m}$ ); c) LIVE/DEAD staining of the cells (green for dead cells, blue for live cells, scale bar $400 \mu \mathrm{m}$ ); d) actin staining of HUVECs inside the device (green for actin, scale bar $200 \mu \mathrm{m}$; inset at 40x). 